EP-32

\title{
Short-term outcome of laparoscopic left lobe liver resection for hepatocellular carcinoma in Vietnam National Cancer Hospital
}

\author{
The Anh PHAM*, Minh Hoang TA, Huy Phuong TRINH
}

Department of Hepatobiliary and Pancreatic Surgery, Vietnam National Cancer Hospital, Ha Noi, Vietnam

Introduction: Hepatocellular carcinoma (HCC) is a common malignant disease in Vietnam with a high mortality rate. The use of laparoscopic liver resection (LLR) has progressively spread in the last 5 years in our country. Althought treatments are dependent primarily on the stages of disease, locations, sizes, and number of tumors. In addition, laparoscopy leads to less post-operative abdominal adhesions and improving operative outcomes.

Methods: Evaluation short-term outcome of patients with diagnosis of HCC who underwent laparoscopic left lobe liver resection totally from August 2018 to August 2019 at our Department.

Results: There were 11 male (84.6\%) and 2 female (15.4\%), with a male to female ratio of 5.5:1; mean age $58.46 \pm 8.67$ years; positive rates fos hepatitis B is 30.8\%; all child's class A cirrhotics; $53.8 \%$ of cases had tumors located in segment 2 and segment 3 and $84.6 \%$ has a single tumor; the mean tumor size is $51.6 \pm 26.7 \mathrm{~mm}$; The operation time is $163.08 \pm 14.94$ minutes; the median postoperative hospital stay is: $7.54 \pm 1.15$ days; no patients had necessity for blood transfusion; the complication rate and mortality rate is $0 \%$.

Conclusions: Laparoscopic left lobe liver resection for HCC is safe and feasible procedure. 